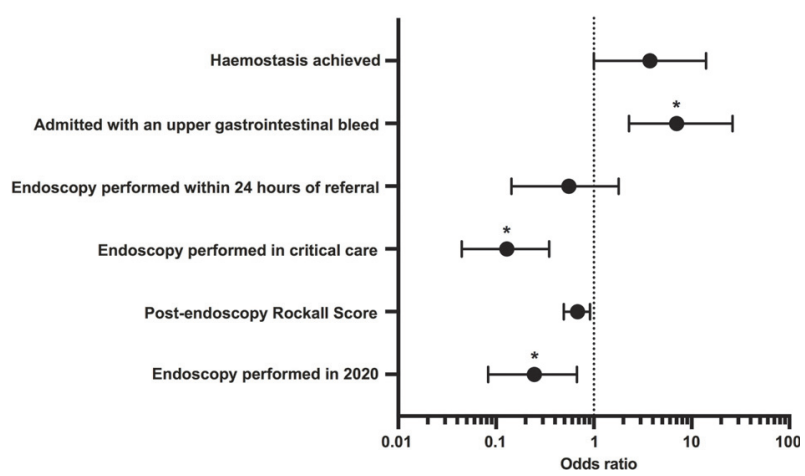

Abstract IDDF2020-ABS-0163 Figure 1 Odds ratio plot demonstrating outcomes of a multiple logistic regression model evaluating risk factors affecting 30-day survival post-endoscopy for UGI bleeds

corresponding dates in 2019. Primary outcome was in-hospital survival at 30 days with secondary outcomes of major rebleeding within 30 days post-procedure and intervention at time of endoscopy.

Results 224 endoscopies for 203 patients with UGI bleeds were included within this study. 19 patients were diagnosed with COVID-19. There was a $44.4 \%$ reduction in the number of procedures performed between 2019 and 2020. Endoscopies performed for UGI bleeds in the COVID-19 era were associated with an adjusted reduced 30-day survival (OR 0.25, 95\% CI 0.08-0.67) (figure 1). There was no increased risk of major re-bleeding or interventions during this era. Patients with COVID-19 did not have adjusted reduced survival or adjusted increased complication rates.

Conclusions Endoscopy for UGI bleeds in the COVID-19 era is associated with reduced survival. No clear cause has been identified but we suspect this is a secondary effect of the response to the COVID-19 pandemic. Urgent work is required to encourage the public to seek medical help if required and to optimise patient pathways to ensure that the best possible patient care is provided.

\section{IDDF2020-ABS-0164 CLINICAL SYMPTOMS, ENDOSCOPIC FINDINGS AND LOWER ESOPHAGEAL SPHINCTER PRESSURE IN PATIENTS WITH ABSENT CONTRACTILITY ON HIGH- RESOLUTION MANOMETRY}

${ }^{1}$ Hang Dao*, ${ }^{2}$ Hue Luu, ${ }^{2}$ Linh Nguyen. ${ }^{1}$ Hanoi Medical University, Vietnam; ${ }^{2}$ Institute of Gastroenterology and Hepatology, Vietnam

\subsection{6/gutjnl-2020-IDDF.104}

Background Absent contractility is a rare esophageal motility disorder, described mainly in patients with systemic diseases. There is no available data on the Asian population as well as in Vietnam. This study aims to describe clinical symptoms, upper gastrointestinal endoscopy findings (UGIE) and lower esophageal sphincter pressure (LES) in patients with absent contractility on high-resolution manometry (HRM).

Methods A cross-sectional study in 67 patients diagnosed with absent contractility on HRM at the Institute of Gastroenterology and Hepatology from March 2018 to April 2019.
Results During the study period, 2762 patients with upper gastrointestinal symptoms were performed HRM in which 67 patients $(2.4 \%)$ were diagnosed with absent contractility on HRM. There is no case with the past history of systemic diseases or diabetes. The female/male ratio was 1.6 , and the mean age was $43.2 \pm 14.8$. Common symptoms were regurgitation (74.6\%), heartburn (28.4\%) and dysphagia (32.8\%). $80.6 \%$ and $44.8 \%$ of the patients had an FSSG and GERDQ score $\geq 8$, respectively. On UGIE, 46\% had reflux esophagitis, mostly Los Angeles grade A. The prevalence of LES $<10$ $\mathrm{mmHg}$ and IRP4s $<5 \mathrm{mmHg}$ were significantly higher in patients with esophagitis on endoscopy.

Conclusions Clinical symptoms of patients with absent contractility were various and nonspecific. The prevalence of low LES pressure was significantly higher in patients having reflux esophagitis.

\section{IDDF2020-ABS-0167 ANXIETY AND DEPRESSION AMONG GASTROENTEROLOGISTS: AN ONLINE SURVEY IN BANGLADESH}

${ }^{1}$ Abdul Mumit Sarkar*, ${ }^{2}$ Madhusudan Saha, ${ }^{3}$ Mushtaque Ahmed Rana, ${ }^{4}$ Shasanka Kumar Saha. 'Rajshahi Medical College, Rajshahi, Bangladesh; ${ }^{2}$ North East Medical College, Sylhet, Bangladesh; ${ }^{3}$ Bangladesh Medical College, Dhaka, Bangladesh; ${ }^{4} \mathrm{M}$ Abdur Rahim Medical College, Dinajpur, Bangladesh

\subsection{6/gutjnl-2020-IDDF.105}

Background Anxiety and depression negatively affect physicians' performance leading to make errors in service providing. Moreover, there is a significant association between professional burnout and anxiety and depression. As a part of this doctor community, gastroenterologists are also at risk of suffering from mental health problems. This study was designed to see the prevalence of anxiety and depression among gastroenterologists in Bangladesh.

Methods Anxiety and depression negatively affect physicians' performance leading to make errors in service providing. Moreover, there is a significant association between professional burnout and anxiety and depression. As a part of this doctor community, gastroenterologists are also at risk of suffering from mental health problems. This study was designed to see the prevalence of anxiety and depression among gastroenterologist in Bangladesh.

Results Out of 166 gastroenterologists, 63 (37.95, mean age $20.2 \pm 8.0$ years, $96.8 \%$ female) responded. The service length of respondents varied from 5 years to 43 years (mean 20.29 \pm 8.09 ) and their working length as specialists varied from 1 to 38 years (mean $13.28 \pm 7.278$ ). In this series symptom scores of $13(20.6 \%)$ and $3(4.8 \%)$ respondents were consistent with borderline anxiety and overt anxiety respectively and symptom scores of $11(17.5 \%)$ and 2 (3.2\%) respondents were consistent with borderline and overt depression respectively. Among them, nine (14.2\%) respondents had both anxiety and depression (including borderline and overt cases). Anxiety was significantly more prevalent (9, 31.0\%) among gastroenterologists of 41-50 years age group $(p=0.007)$ and those are working as specialists less than or equal to five years $(6 ; 75 \%),[\mathrm{P}=0.014]$. Depression was also more common among gastroenterologists of age $41-50$ years group $7(24.13 \%)$, doing government service 10 (19.60\%), service length $\leq 15$ years 5 (29.41), and working as specialist $\leq 10$ years 7 (31.82\%), but the difference was not significant. 
Conclusions Anxiety and depression among gastroenterologists are more prevalent in comparison to the general population. Gastroenterologist of younger age group, working in the government sector, less experience in working as a specialist, are more vulnerable. Adequate opportunities should be created for young gastroenterologists to increase their professional skills.

\section{IDDF2020-ABS-0170 THE CLINICAL CHARACTERISTICS AND OUTCOMES OF CROHN'S DISEASE PATIENTS WITH PERIANAL DISEASE ONSET AT PEDIATRIC VERSUS ADULT: A 10-YEAR OBSERVATIONAL STUDY}

${ }^{1}$ Haichao Wang*, ${ }^{1}$ Yalin Wu, ${ }^{2}$ Chen Ye, ${ }^{1}$ Zhanju Liu. ${ }^{1}$ Department of Gastroenterology, The Shanghai Tenth People's Hospital, Tongji University, China; ${ }^{2}$ Medical College of Soochow University, China

\subsection{6/gutjnl-2020-IDDF.106}

Background Perianal disease (PD) is a common complication in Crohn's disease (CD) patients. However, significances of $\mathrm{PD}$, regarding the onset-age, are undefined in perianal Crohn's disease (PCD). We aim to compare PCD patients with pediatric-onset PD (POP) and adult-onset PD (AOP) in terms of their natural history for over 10 years.

Methods Medical records of 293 PCD patients in the Tenth People's Hospital affiliated with Tongji University (Shanghai, China) from January 2008 to December 2017 were reviewed retrospectively. The clinical characteristics and outcomes of patients with POP $(n=84)$ and AOP $(n=209)$ were assessed.

Results Among 293 PCD patients, the mean PD onset-age was 25.9 years. Frequencies of complex perianal fistulas $(71.7 \%$ vs $50.0 \%, p=0.011)$ and infliximab treatment $(33.3 \%$ vs $22.0 \%, p=0.044)$ in POP group were higher than those in AOP group. Medians (interquartile range) diagnostic delay for POP and AOP groups were 12 (2-48) and $24(2.5-60)$ months, respectively. Higher percentage of structuring behavior $(42.1 \%$ vs $27.4 \%, p=0.024)$, current smoking status $(12.9 \%$ vs $4.8 \%, p=0.04)$, and abdominal surgery $(21.1 \%$ vs $4.8 \%, p=0.001)$ were observed in AOP group. Furthermore, structuring behavior was associated with developing PD in adulthood (odds ratio: 2.029, 95\% confidence interval: $1.143-3.604, p=0.016$ ). More recurrence of PD was found in the patients with AOP after withdrawing of infliximab treatment $(38.9 \%$ vs $0 \%, p=0.024)$. The cumulative probabilities of abdominal surgery in POP group were lower than those of in AOP group $(p=0.007)$. Increased use of infliximab was associated with decreased rate of abdominal surgery in patients with AOP $(r=-0.900$, $p=0.037$ ).

Conclusions AOP may indicate more unfavorable outcomes than POP. Those patients with AOP require early and longterm IFX treatment due to their complicated behaviors.

\section{IDDF2020-ABS-0173 EFFICACY AND SAFETY OF THE ENDOSCOPIC RESECTION OF 10- TO 20- MM NON-PEDUNCULATED COLORECTAL POLYPS: A SYSTEMATIC REVIEW AND POOLED ANALYSIS}

${ }^{1}$ Xin Yuan*, 'Zhixin Zhang, ${ }^{2}$ Jiarong Xie, ${ }^{2} Y u$ Zhang, ${ }^{2}$ Hongpeng Lu, ${ }^{2}$ Weihong Wang, ${ }^{2}$ Lei Xu. ${ }^{1}$ College of Medicine, Ningbo University, China; ${ }^{2}$ Department of Gastroenterology, Ningbo First Hospital, China

\subsection{6/gutjpl-2020-IDDF.107}

Background We performed this systematic review and pooled analysis to assess the effectiveness and safety of different endoscopic resection methods for 10 - to 20 -mm non-pedunculated colorectal polyps.

Methods Articles in PubMed, EMBASE, and the Cochrane Library related to the common endoscopic treatment of 10to $20-\mathrm{mm}$ non-pedunculated polyps published as of April 2020 were searched. Primary outcomes were the complete resection rate (CRR) and the en bloc resection rate (EBRR).

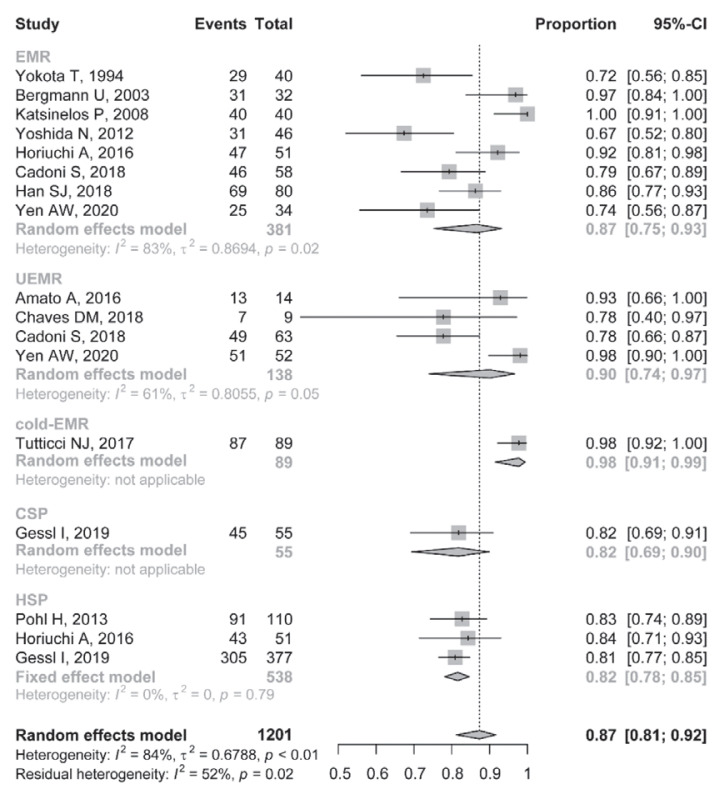

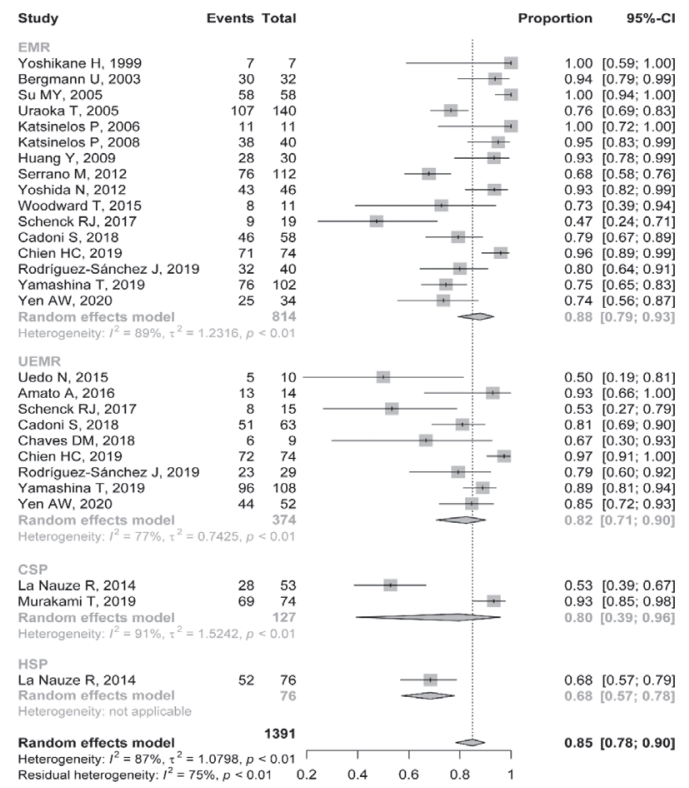

Abstract IDDF2020-ABS-0173 Figure 1 Forest plots reporting the CRRs and EBRRs of different types of resection method 